\title{
Signs of Impending Birth
}

National Cancer Institute

\section{Source}

National Cancer Institute. Signs of Impending Birth. NCI Thesaurus. Code C92881.

The indicating characteristics in a pregnant mother who is in the process of labor and delivery of a fetus; common signs and symptoms include nesting, anxiety, excitement, loss of mucous plug, increased urinary frequency and loose bowel movements. 